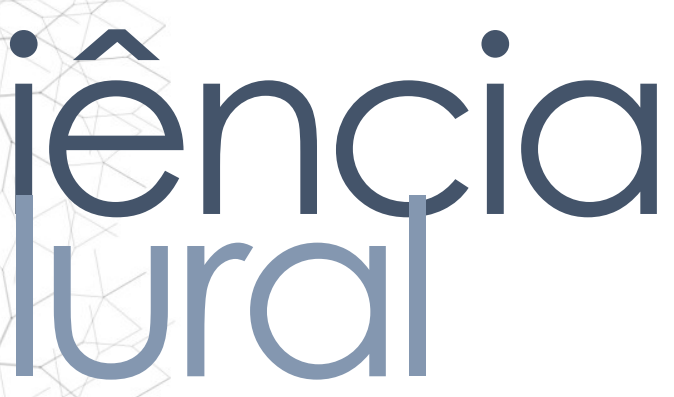

\title{
CONTRIBUIÇÕES DA VIVÊNCIA EM COMUNIDADE PARA FORMAÇÃO ACADÊMICA EM FISIOTERAPIA
}

Contributions of a community experience to academic training in physiotherapy

Contribuciones de la experiencia comunitaria a la formación académica en fisioterapia

Arlon Néry do Nascimento • Fisioterapeuta pela Cristo Faculdade do Piauí CHRISFAPI • E-mail: arlonnerys3@gmail.com

Amanda Maria Brito da Silva • Fisioterapeuta pela Cristo Faculdade do Piauí

CHRISFAPI • E-mail: amanndabrito@hotmail.com

Carolyne Carvalho Caxias • Fisioterapeuta pela Cristo Faculdade do Piauí CHRISFAPI • E-mail: carolynec.caxias@gmail.com

Dionatan Christophe Alves de Paula • Fisioterapeuta pela Cristo Faculdade do Piauí CHRISFAPI • E-mail: diochristophe@gmail.com

Mayane Carneiro Alves Pereira • Fisioterapeuta pela Universidade Federal do Piauí (UFPI) • Mestre em Ciências e Saúde pela UFPI • Fisioterapeuta da Fundação Municipal de Saúde de Piripiri-PI • Docente em Saúde Pública da Cristo Faculdade do Piauí (CHRISFAPI) • E-mail: mayanealves@hotmail.com

Autor correspondente:

Arlon Néry do Nascimento • E-mail: arlonnerys3@gmail.com 


\section{RESUMO}

Introdução: Durante sua formação, o fisioterapeuta é preparado para atuar em todos os níveis de atenção, não ficando restritos somente às ações reabilitadoras e curativas às quais são comumente associados. A promoção e educação em saúde, fazem parte de suas atribuições e visam a autonomia na saúde da população. Objetivo: Relatar a experiência de acadêmicos de um curso de Fisioterapia durante a vivência no estágio supervisionado no setor de saúde na comunidade. Metodologia: Os relatos apresentados neste estudo estão vinculados à atuação de quatro discentes do curso de Fisioterapia em uma Unidade Básica de Saúde do município de Piripiri-PI, durante o período de fevereiro a março do ano de 2020. As atividades eram realizadas três vezes por semana, ficando estabelecido a divisão em três momentos (Alongamento e aferição da pressão arterial; Dinâmica; Roda de conversa), com o intuito de trazer uma melhor interação da comunidade com os acadêmicos, proporcionar ganhos de saúde aos participantes e promover um maior conhecimento sobre os temas abordados. Resultados: Essas ações realizadas durante o estágio, resultaram na construção de um vínculo entre os acadêmicos e a comunidade gerando promoção e educação para ambos os lados, bem como a desconstrução da sociedade sobre as atribuições do fisioterapeuta. Conclusões: Desta forma finda-se que o estágio na comunidade agrega ao conhecimento teórico e prático dos acadêmicos uma experiência rica, que leva ao desenvolvimento de habilidades e trabalho em equipe, assim como proporciona uma maior valorização sobre a importância da atenção primária e o papel do fisioterapeuta na equipe multidisciplinar.

Palavras-Chave: Comunidade; Saúde; Fisioterapia; Estágio.

\section{ABSTRACT}

Introduction: During their training, the physiotherapist is prepared to work at all levels of care, not being restricted only to the rehabilitation and corrective actions to which they are commonly associated. Health promotion and education, it is part of their duties and aim at the autonomy in the health of the population. Objective: To report the experience of a physiotherapy course's academics during their supervised internship in the community health sector. Methodology: The reports presented in this study are linked to the performance of four students of the Physiotherapy course in a Basic Health Unit in the municipality of Piripiri-PI, between February and March of 2020. The activities were carried out three times a week, the division was established in three moments (Stretching and blood pressure measurement; Dynamic; Conversation circle) to bring a better interaction between the community and academics, providing health gains to participants and promoting more excellent knowledge on the topics addressed. Results: These actions carried out during the nternship resulted in the construction of a bond between academics and the ommunity, generating promotion and education for both sides and the econstruction of society about the physiotherapist's duties. Conclusions: Thus, it ds that the internship in the community adds to the theoretical and practical owledge of academics a rich experience, which leads to the development of skills 
and teamwork, as well as providing a greater appreciation of the importance of primary care and the role of the physiotherapist. in the multidisciplinary team.

Keywords: Community. Health. Physiotherapy. Phase.

\section{RESUMEN}

Introducción: Durante su formación, el fisioterapeuta se prepara para trabajar en todos los niveles asistenciales, no limitándose únicamente a las acciones rehabilitadoras y correctoras a las que comúnmente se les asocia. La promoción y educación para la salud, forma parte de sus funciones y tiene como objetivo la autonomía en la salud de la población. Objetivo: Relatar la experiencia de los académicos de un curso de fisioterapia durante sus prácticas supervisadas en el sector de la salud comunitaria. Metodología: Los informes que se presentan en este estudio están vinculados a la actuación de cuatro estudiantes del curso de Fisioterapia en una Unidad Básica de Salud del municipio de Piripiri-PI, entre febrero y marzo de 2020. Las actividades se realizaron tres veces por semana, la división se estableció en tres momentos (Estiramiento y medición de la presión arterial; Dinámica; Círculo de conversación) para traer una mejor interacción entre la comunidad y los académicos, proporcionando ganancias de salud a los participantes y la promoción de un conocimiento más excelente sobre los temas abordados. Resultados: Estas acciones realizadas durante la pasantía resultaron en la construcción de un vínculo entre los académicos y la comunidad, generando promoción y educación para ambas partes y la deconstrucción de la sociedad sobre las funciones del fisioterapeuta. Conclusiones: Así, se concluye que la pasantía en la comunidad agrega a los conocimientos teóricos y prácticos de los académicos una rica experiencia, que lleva al desarrollo de habilidades y al trabajo en equipo, además de proporcionar una mayor valoración de la importancia de la atención primaria y del rol del fisioterapeuta. en el equipo multidisciplinario.

Palabras clave: Comunidad. Salud, fisioterapia. Prácticas. 


\section{Introdução}

A Atenção Primária à Saúde (APS) constitui a porta de entrada aos serviços de saúde pública, sendo caracterizada por um conjunto de ações, no âmbito coletivo e individual, que compreende a promoção e prevenção à saúde, prevenção de agravos, diagnóstico, tratamento e reabilitação ${ }^{1}$. Todas as ações são orientadas pelos princípios da universalidade, acessibilidade e da coordenação ao cuidado, responsabilização, integralidade, humanização, vínculo e continuidade, equidade e participação social².

Durante sua formação acadêmica, o profissional fisioterapeuta é preparado para atuar em todos os níveis de atenção, não ficando restritos somente às ações reabilitadoras e curativas às quais são comumente associados. O olhar sobre o fisioterapeuta como meramente profissional reabilitador, durante muito tempo, o excluiu da rede básica, dificultando o acesso da população aos serviços de fisioterapia e impossibilitando o profissional de trabalhar na APS. Nos últimos anos, passou a existir uma preocupação maior para que esses profissionais adquiram habilidades e competências para atuação na APS 3 .

A promoção e educação em saúde, faz uso de metodologias ativas que visem a autonomia na saúde da população, elaboração de cartilhas informativas e desenvolvimento de projetos sociais preventivos estão dentre as principais competências do fisioterapeuta na APS. Essas atividades podem ser realizadas de forma individual ou em grupos, e atuar sobre as diferentes demandas de grupos como aqueles direcionados a idosos, gestantes, hipertensos e diabéticos, portadores de hanseníase, usuários de prótese e órtese, trabalhadores vítimas de acidentes e doenças do trabalho, grupos de orientação postural, com mães que possuem filhos com problemas neurológicos ou outros acometimentos e até mesmo trabalhos em creches 4 .

É necessário incentivar as ações na APS desde o início da formação profissional, para que elas fortaleçam a relação entre prática e teoria, oportunizando aos acadêmicos um espaço de construção do conhecimento, em atuação articulada com o serviço e, uso imaginação e trabalho em equipe para o desenvolvimento de tarefas ${ }^{5}$.

Dentro deste contexto, observa-se que a presença do fisioterapeuta na punidade é essencial para a melhoria da qualidade de vida da população, além o proporciona aprendizados e experiências enriquecedoras para o profissional. Em 
decorrência dessa mudança de paradigma, instituições de ensino superior introduziram em sua grade curricular disciplinas voltadas para a saúde pública além de proporcionarem aos acadêmicos um estágio na comunidade.

Com base no exposto, o presente artigo objetiva relatar a experiência de acadêmicos de um curso de Fisioterapia durante a vivência no estágio supervisionado no setor de saúde na comunidade.

\section{Metodologia}

Trata-se de um estudo descritivo, do tipo relato de experiência que se refere a um tipo de pesquisa em que há a construção do conhecimento baseado no território qualitativo, de acordo com a reprodução ativa de uma vivência, elaborando-se relatos de memória, no qual os sujeitos presentes tem o objetivo de estender e assimilar os saberes adquiridos na experiência ${ }^{6}$.

Os relatos estão vinculados à atuação de quatro discentes do curso de Fisioterapia de uma Faculdade do Norte do Piauí, o campo de atuação ocorreu no território de atuação de uma Unidade Básica de Saúde (UBS) da zona urbana do município de Piripiri-PI, durante o período de estágio curricular (fevereiro a março do ano de 2020). As ações realizadas voltaram-se para a população residente no bairro do referido território (UBS 17).

Os estagiários eram supervisionados por um preceptor, o número de participantes na comunidade foi afetado no decorrer do ciclo, devido a eventos climáticos e, por fim, com o início do distanciamento social voluntário em virtude da Pandemia causada pelo novo coronavírus COVID-19.

Antes do início das atividades com a comunidade, foram realizadas reuniões entre os discentes e o docente responsável pelo estágio, com o intuito de organizar as ações e esclarecer dúvidas sobre o público alvo, neste momento, foram estabelecidas datas dos encontros e os temas a serem debatidos. O estágio foi organizado em clos, cada um era formado por seis estagiários, no ciclo de comunitária os mesmos se bdividiram em três duplas, ficando responsáveis por desempenhar uma atividade 
em específico, abordando o tema em questão a cada dia. No Fluxograma 1 pode se observar como foram dividas as tarefas entre os acadêmicos.

Fluxograma 1 - Divisão das equipes e tarefas para o desenvolvimento das atividades semanais, UBS 17. Piripiri-PI, 2020.

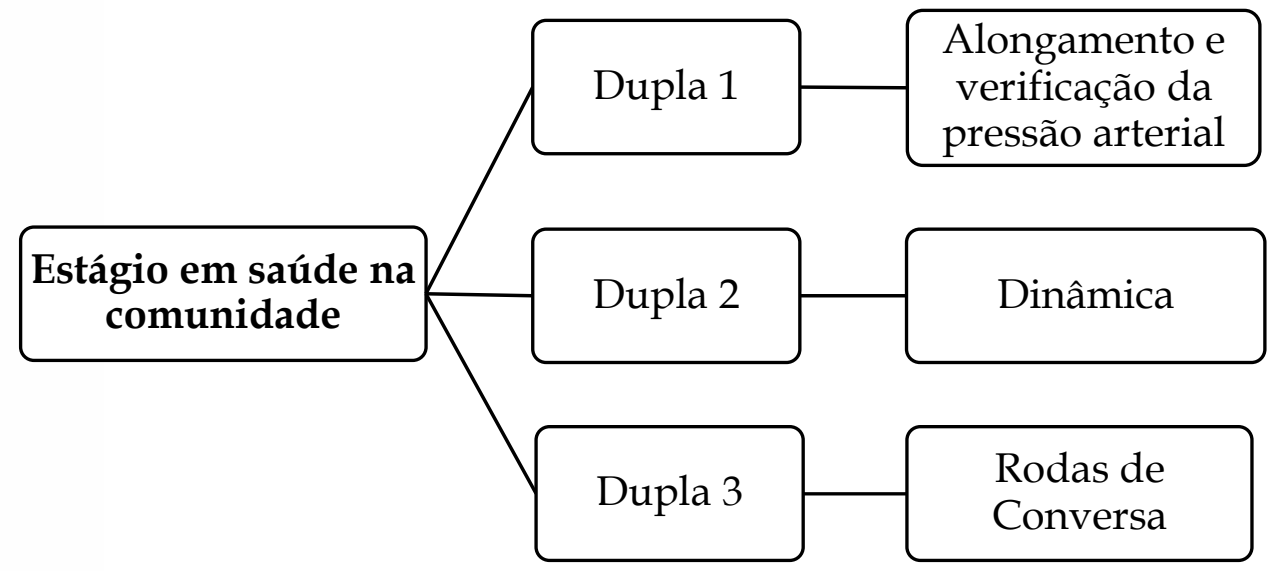

Fonte: Elaborado pelos autores, 2020.

As atividades de prevenção e promoção de saúde na comunidade foram desenvolvidas em uma instituição religiosa que se encontra dentro do território, uma vez que o espaço já era utilizado na comunidade para ações conjuntas. Vale ressaltar que a execução das ações dependia de diversos fatores como o tempo (clima), quantidade de participantes, nível de conhecimento do público, entre outros. Diante disso, é se suma importância manter esse processo dinâmico e passível de mudanças quando necessário, para se adequar às necessidades da população.

A APS é construída com um conjunto de ações de saúde, nos âmbitos coletivos e individuais, envolvendo cuidados em promoção, proteção e educação em saúde, dirigidos a populações de territórios definidos com diferentes temáticas de acesso a essa informação e as ações voltadas com adaptações ao ambiente7.

O público abordado nessas dinâmicas era majoritariamente mulheres idosas, o que vai de encontro a estudos encontrados na literatura, na qual afirmam que mulheres com faixa etária entre 65 e 69 anos têm um envelhecimento com maior nvolvimento social ${ }^{8}$. As atividades desenvolvidas no estágio tinham como intuito bordar a cada dia temas diferentes com rodas de conversa, demonstrações de tazes, objetos, simulações de situações, dinâmicas e relatos de experiência dos 
participantes. Para obter interação e qualidade de vida da população é recomendável implementar programas de intervenção comunitária dinâmicos e educativos, em particular envolvendo atividade física9 9

As atividades eram realizadas três vezes por semana, ficando estabelecido a divisão em três momentos, na qual cada dupla ficava responsável por uma tarefa no dia e outro dia alternava-se a tarefa, de modo que todas as duplas realizassem todas as atividades propostas no decorrer do ciclo. A pressão arterial (PA) de todos os indivíduos era verificada após cinco minutos de repouso a partir do momento de chegada no local e no final das atividades.

Para as rodas de conversa, foram abordados temas relacionados à educação em saúde pertinentes a realidade da população adscrita, com o objetivo de prevenção a condições específicas e promoção à saúde, sendo incentivada a interação e troca de experiências sobre os temas abordados a cada dia, como preconiza a Política de Educação Popular em Saúde. Por meio dessas ações, procurou-se relacionar o tema debatido à dinâmica que seria aplicada, reforçando a compreensão e realização das práticas sugeridas durante a explanação. No quadro 1 pode-se observar as atividades e temas discutidos nas rodas de conversa.

Quadro 1 - Atividades de prevenção e promoção em saúde e temas debatidos nas rodas de conversa no setor de comunitária, UBS 17. Piripiri-PI, 2020.

\begin{tabular}{|l|l|}
\hline ATIVIDADES & TEMAS \\
\hline Treino de Marcha & Câncer \\
\hline $\begin{array}{l}\text { Exercícios Físicos com materiais adaptados no } \\
\text { ambiente }\end{array}$ & Diabetes \\
\hline Dinâmica de agilidade & Hipertensão \\
\hline Dança & Sexualidade na Terceira Idade \\
\hline Dinâmica de interação social & Saúde Mental \\
\hline Treino Funcional & Importância do Exercício Físico \\
\hline Treino de coordenação e equilíbrio & Prevenção de Quedas \\
\hline
\end{tabular}




\begin{tabular}{|l|l|}
\hline Exercícios para musculatura do assoalho pélvico & Incontinência Urinária \\
\hline Dinâmica com grupo alimentar & Cuidados com Alimentação \\
\hline
\end{tabular}

Fonte: Elaborado pelos autores, 2020.

Como forma de discussão das experiências vividas pelos estagiários era realizado diariamente, após as atividades com a comunidade, uma discussão entre os estagiários e o preceptor. Após o debate era organizado um diário de campo, no qual os estagiários descreviam suas vivências, casos clínicos, métodos de pesquisa, aplicabilidade de técnicas de avaliação e protocolos de atendimentos de forma detalhada, além de expectativas e relatos reais sobre a experiência vivenciada dentro do ciclo de comunidade.

O diário de campo é como um instrumento para desenvolvimento de pesquisa e aprendizado de sucesso, por se tratar de uma metodologia que permite aos pesquisadores descreverem pessoas, objetos, acontecimentos, lugares, atividades, diálogos, ideias, estratégias, reflexões e palpites. Dando ênfase às especulações sentimentos, impressões, problemas e as ideias dos pesquisadores, além de seus planos para futuras considerações ${ }^{\mathbf{1 0}}$.

As atividades realizadas tiveram o intuito de trazer uma melhor interação da comunidade com os acadêmicos, proporcionar ganhos de saúde os participantes e promover um maior conhecimento sobre os temas abordados. Durante as atividades, os discentes utilizavam linguagem clara, simples e informal dispondo de materiais de baixo custo e alguns de fabricação própria para auxiliar na explanação dos temas abordados.

\section{Resultados e discussão}

\section{ormação Acadêmica em Fisioterapia}

A formação acadêmica em fisioterapia permite o desenvolvimento de uma ducação voltada para a APS secundária e terciária, com ênfase na reabilitação de doenças, o que leva o profissional a uma limitação no que diz respeito às ações 
voltadas para APS, que é relacionada à promoção e proteção da saúde e a prevenção de agravos da população.

Um estudo que descreve o perfil do fisioterapeuta atuante na APS relata que esta formação está em constante processo de transformação. Existe um interesse das instituições de possibilitar experiências aos acadêmicos a fim de aumentar sua perspectiva, possibilitando maiores possibilidades no futuro profissional em todos os níveis de atenção, indo além apenas da reabilitação ${ }^{\mathbf{1 1}}$.

Durante o estágio obrigatório, o acadêmico vivencia várias etapas, que são divididas em ciclos, de acordo com o regulamento de cada instituição e, normalmente, são realizadas no ambiente hospitalar ou em clínicas, com ênfase nas ações curativas e também há o ciclo de saúde comunitária, que acontece com o auxílio de uma equipe multiprofissional da Estratégia Saúde da Família (ESF) do bairro em que a instituição está vinculada.

$\mathrm{O}$ ensino acadêmico e as práticas dos profissionais devem incluir $\mathrm{o}$ desenvolvimento das ações preventivas e curativas, relacionadas anteriormente aos processos de adoecimento e estruturada com base em especialidades e, agora, pautada também no saber popular e na demanda local, respeitando suas peculiaridades e diferentes práticas de cura12. Entende-se que esse processo leva tempo e demanda de uma maior comunicação entre os profissionais que atuam nas redes de atenção à saúde e que a busca por uma visão integral aos cuidados prestados, aliando-se às tecnologias necessárias e a participação ativa da comunidade, contribuem para o aprimoramento da assistência em saúde.

\section{Caracterização do Cenário da Prática}

No estágio realizado na comunidade, preconizam-se ações voltadas para a prevenção e promoção de saúde na comunidade, com a finalidade de promover uma melhor qualidade de vida aos usuários e um envelhecimento mais saudável. A população adscrita no território de prática e usuária frequente da UBS é composta incipalmente por idosos que possuem, entre as principais comorbidades, diabetes e 
hipertensão arterial sistêmica (HAS) e já são previamente acompanhados pelos profissionais da UBS.

Nas últimas décadas, a APS direcionada para o processo do envelhecimento tem aumentado, em virtude do aumento da longevidade da população mundiali3 ${ }^{13}$ Isso demonstra um desafio para os responsáveis por levar o conhecimento a esses usuários, pois a maior compreensão das doenças mais prevalentes e os mecanismos que os envolvem pode possibilitar um maior autocuidado e autonomia nas tomadas de decisão em saúde, com essa prática, os idosos podem trocar experiências e ampliar o saber coletivo.

O planejamento realizado pela equipe, composta por dois preceptores e seis estagiários e subdividida em duplas supervisionadas e responsáveis por cada ação, que foi estabelecida de acordo com a elaboração de um cronograma com todas as atividades semanais e diárias, baseando as atividades propostas nas temáticas mais relevantes para os usuários, estabelecendo uma dinâmica de rodas de conversas e atividades físicas por meio de circuitos, com o intuito de levar a uma maior interação e adesão da atividade proposta.

Um estudo realizado na Paraíba que analisou a organização e planejamento de ações individuais e conjuntas entre as equipes da atenção básica e as equipes do Núcleo Ampliado de Saúde da Família (NASF) demonstra a importância do planejamento e pactuação inicial do trabalho a ser desenvolvido pelas equipes, mas evidencia também a necessidade deste processo de planejamento ser dinâmico e sistemático, de tal forma que mantenha as pactuações vivas e úteis às demandas cotidianas do processo de trabalho entre as equipes ${ }^{\mathbf{1 4}}$.

\section{Saúde na Comunidade: Reflexões, Vivências e Aprendizados no Estágio}

A princípio, o contato com a comunidade demanda dos acadêmicos um maior poder de interação, visto que é fundamental para que o público, em sua maioria de dosos, tenha uma maior adesão ao que foi proposto para as atividades. Além disso, a

inguagem que anteriormente é técnica deve ser adaptada para um nível em que todos 
consigam entender e deixar o ambiente confortável as trocas de experiências sobre o tema debatido, com cuidado para não fugir do cerne da questão em pauta.

Um estudo que analisou a contribuição do Programa de Educação pelo Trabalho em Saúde - PETSaúde/GraduaSUS na formação dos Fisioterapeutas, evidenciou que a vivência em um ambiente cotidiano e prático das UBS, leva a uma ampliação do conceito de saúde, da associação dos fatores sociais no processo saúde-doença, assim como de integralidade da assistência ${ }^{15}$.

Com a escassez de materiais necessários para a realização de atividades práticas, a busca por novos métodos e a criatividade ganha um novo espaço dentro da preparação das atividades, tornando o ambiente comunitário um maximizador dos conhecimentos adquiridos durante a formação, o que torna o trabalho em equipe uma ferramenta indispensável para ações bem sucedidas.

A troca de saberes que acontece entre a população e os estagiários é outro fator que enriquece a experiência do acadêmico e leva ao desenvolvimento de uma visão sobre humanização em saúde na prática, pois possibilita a construção de um comportamento ético durante o acompanhamento das condições de saúde, estimulando a formulação conjunta de conhecimentos, possibilitados pelas ações em saúde, além da construção de vínculo acadêmico-paciente, acadêmico- acadêmico e acadêmico-supervisor. O estágio curricular é o momento em que o aluno experimenta as vivências em saúde e se defronta com situações de conflitos, disputas, tensões, relações de poder e outros acontecimentos inusitados que possam surgir ${ }^{16}$. Uma vez que o aprender na formação, especialmente no ambiente prático, possibilita a assimilação e partilha de conhecimentos por toda a equipe e pacientes.

Essa vivência permite aos estudantes uma reorientação sobre a futura atuação profissional, partindo do ponto de vista de que o usuário em saúde vai além de uma doença a ser tratada, levando em consideração que ele é um agente ativo no processo da saúde e que o contexto no qual está inserido e sua visão são importantes e devem azer parte da tomada de decisão. Também propicia o surgimento de novas rspectivas para a Fisioterapia como agente importante na prevenção e educação em de nas comunidades. 


\section{Conclusões}

O estágio na comunidade agrega ao conhecimento teórico e prático dos acadêmicos uma experiência rica que leva ao desenvolvimento de habilidades e trabalho em equipe, assim como proporciona uma maior valorização sobre a importância da atenção primária e o papel do fisioterapeuta na equipe multidisciplinar que atua neste meio. Destaca-se, nesse sentido, que deve haver uma alteração na metodologia empregada pelas instituições de ensino, tornando indispensável a realização de ações nas quais o estudante deve vivenciar como é realizado o acompanhamento de famílias em uma comunidade nos anos iniciais de sua formação acadêmica.

\section{Referências}

1. Ministério da Saúde (Brasil). Atenção Básica. 2017 maio 18. [acesso em 2020 maio 17]. Disponível em: https://www.saude.gov.br/artigos/770-sistema-nacional-desaude/40315-atencao-basica.

2. Miranda GBN, Teixeira RC. Atuação do fisioterapeuta na atenção primária: conhecimento dos acadêmicos do último semestre. Cad Educ. Saúde Fisioter. 2014; 1(2):13-25.

3. Ribeiro CD, Flores-Soares MC. Desafios para a inserção do fisioterapeuta na atenção básica: o olhar dos gestores. Rev. Salud Pública. 2015; 1(3): 379-393.

Disponível em: Desafios para a inserção do fisioterapeuta na atenção básica: o olhar dos gestores.

4. Morais RA, Evangelista AR, Oliveira $\mathrm{ACB}$, et al. O papel da fisioterapia na atenção básica: revisão sistemática de literatura. EEDIC. 2017; 4(1): 1-6. Disponível em: https:/ / pdfs.semanticscholar.org/2b86/ba2f2c30c7363c3919316828d5b7d9b4b7dd.p df.

Massote AW, Belisário AS, Gontijo ED. Atenção primária como cenário de prática na percepção de estudantes de medicina. Rev. Bras. Educ. Med. 2011[acesso em 2020 un 01]; 35(4): 445-453. Disponível em:

https://www.scielo.br/pdf/rbem/v35n4/a02v35n4.pdf.

Drauto, MR, Faria AA. Relato de experiência: Uma narrativa científica na pósdernidade. Estud. pesqui. psicol. 2019. [acesso em 2020 dez 10]; 19 (1): 223-237. 
Disponível em: http:/ / pepsic.bvsalud.org/scielo.php?script=sci_arttext\&pid=S180842812019000100013.

7. Gauer APM, Ferretti F, Teo CRPA, et al. Ações de reorientação da formação profissional em Fisioterapia: enfoque sobre cenários de prática. Interface (Botucatu). 2018 abr/jun [acesso em 2020 jun 01]; 22(65): 565-576. Disponível em: https:/ / doi.org/10.1590/1807-57622016.0852.

8. Neri AL, Vieira LAM. Envolvimento social e suporte social percebido na velhice. Rev. Bras. Geriatr. Gerontol.. 2013 [acesso em 2020 jun 01]; 16(3): 419-432. Disponível em: https:/ / doi.org/10.1590S1809-98232013000300002.

9. Bastos MAMSC, Monteiro JMMP, Faria CMGM, et al. Participação em programas de intervenção comunitária e qualidade de vida: resultados de um estudo multicêntrico em Portugal. Rev. Bras. Geriatr. Gerontol. 2020 [acesso em 2020 jun 01]; 23(6): 1-14. Disponível em: https:/ / doi.org/10.1590/1981-22562020023.190017.

10. Afonso T, Silva SSC, Pontes FAR, et al. O uso do diário de campo na inserção ecológica em uma família de uma comunidade ribeirinha amazônica. Psicol. Soc. 2015 [acesso em 2020 jun 01]; 27(1): 131-141. Disponível em:

http:/ / www.scielo.br/scielo.php?script=sci_arttext\&pid=S010271822015000100131\& lng=en\&nrm=iso.

11. Fernandes SCS, Ros MA. Desconstruir para transformar: o perfil do fisioterapeuta para o Núcleo de Apoio à Saúde da Famúlia. Fisioter. Bras. 2018 [acesso em 2020 maio 11]; 19(2): 249-258. Disponível em: http://fi-

admin.bvsalud.org/document/view/va5q3.

12. Pedroso RT, Nogueira CAG, Damasceno CN, et al. A Educação Baseada na Comunidade no Ensino Médico na Uniceplac (2016) e os Desafios para o Futuro. Rev. Bras. Educ. Med. 2019 [acesso em 2020 maio 31]; 43(4): 117-130. Disponível em: http:/ / www.scielo.br/scielo.php?script=sci_arttext\&pid=S010055022019000400117\& $\operatorname{lng}=\mathrm{en} \& \mathrm{nrm}=$ iso.

13. Stobaus CD, Lira GA, Ribeiro KSQS. Elementos para um envelhecimento mais saudável através da promoção da saúde do idoso e educação popular. Estud. Interdiscip. Envelhec. 2018 [acesso em 2020 maio 30]; 23(2): 25-49. Disponível em: https:/ / pesquisa.bvsalud.org/ripsa/resource/pt/biblio-1010003.

14. Silva ICB, Silva LAB, Lima RSA, et al. Processo de trabalho entre a Equipe de Atenção Básica e o Núcleo de Apoio à Saúde da Família. Rev. Bras. Med. Fam. Comunidade. 2017 [acesso em 2020 maio11]; 12(39): 1-10. Disponível em: https:/ /rbmfc.org.br/rbmfc/article/view/1433/857.

Viana SBP, Tomaz GGA, Manerich LS. Contribuição do pet-saúde graduasus na mação de estudantes de fisioterapia. Humanidades \& Inovação. 2020 [acesso em 


\section{ciência plựl}

2020 maio 30]; 7(6). Disponível em:

https://revista.unitins.br/index.php/humanidadeseinovacao/article/view/2540.

16. Silva Júnior AP, Oliveira B, Aparecido A. Estágio curricular supervisionado em Educação Física: aproximações com a teoria de Norbert Elias. Actualid. Investig. Educ. 2018 [acesso em 2020 jun 01]; 18(3): 681-701. Disponível em: https://doi.org/10.15517/aie.v18i3.34433. 